\title{
Extracorporeal membrane oxygenation support in pediatrics
}

\author{
Tugba Erdil ${ }^{1,2}$, Frithjof Lemme ${ }^{1,2}$, Alexander Konetzka ${ }^{1,2}$, Anna Cavigelli-Brunner ${ }^{2,3}$, Oliver Niesse $^{2,3}$, \\ Hitendu Dave ${ }^{1,2}$, Peter Hasenclever ${ }^{1,2}$, Michael Hübler ${ }^{1,2}$, Martin Schweiger ${ }^{1,2}$ \\ ${ }^{1}$ Pediatric Cardiovascular Surgery, Pediatric Heart Center, Department of Surgery, University Children's Hospital Zurich, Zurich, Switzerland; \\ ${ }^{2}$ Children's Research Centre, University of Zurich, Zurich, Switzerland; ${ }^{3}$ Pediatric Cardiology, Pediatric Heart Center, Department of Surgery, \\ University Children's Hospital Zurich, Zurich, Switzerland \\ Correspondence to: Martin Schweiger, MD, PD. Division of Congenital Cardiovascular Surgery, University Children's Hospital Zurich, Zurich, \\ Switzerland. Email: martin.schweiger@kispi.uzh.ch.
}

\begin{abstract}
Extracorporeal membrane oxygenation (ECMO) is a general term that describes the short- or long-term support of the heart and/or lungs in neonates, children and adults. Due to favorable results and a steady decline in absolute contraindications, its use is increasing worldwide. Indications in children differ from those in adults. The ECMO circuit as well as cannulation strategies also are individualized, considering their implications in children. The aim of this article is to review the clinical indications, different circuits, and cannulation strategies for ECMO. We also present our institutional experience with 92 pediatric ECMO patients (34 neonates, 58 pediatric) with the majority $(80 \%)$ of veno-arterial placements between 2014 until 2018. We further to also highlight ECMO use in the setting of cardiac arrest [extracorporeal cardiopulmonary resuscitation (CPR) or eCPR].
\end{abstract}

Keywords: Extracorporeal membrane oxygenation (ECMO); extracorporeal cardiopulmonary resuscitation (eCPR); pediatrics

Submitted Jun 29, 2018. Accepted for publication Aug 23, 2018.

doi: 10.21037/acs.2018.09.08

View this article at: http://dx.doi.org/10.21037/acs.2018.09.08

\section{Introduction}

Extracorporeal membrane oxygenation (ECMO) is a lifesaving tool and substantial part of every mechanical circulatory support program. There are substantial differences in ECMO in pediatric patients compared to adults, including indications, setup of the ECMO circuit, as well as implantation sites. The Extracorporeal Life Support Organization (ELSO) represents the largest database worldwide and produces regular reports on international growth, outcomes, complications, and technology used in ECMO. Traditionally, there is one annual report focusing on adults and another one on pediatrics. The latest ELSO report showed that $71 \%$ of all ECMO implants were done in children, and neonates represented $47 \%$ of the whole cohort (1). There are almost 60,000 children receiving ECMO support who have been reported to the ELSO registry.

Recent advances in ECMO have led to increasing interest in its use during cardiopulmonary resuscitation (CPR).
However, decisions regarding extracorporeal CPR (eCPR) in children are difficult because of the current paucity of robust data. This article aims to provide a short overview of indications for ECMO in the pediatric population, highlight differences between pediatric and adult ECMO, and share our own results, with a focus on eCPR.

\section{Indications}

Traditionally, pediatric ECMOs were used in congenital heart surgery when weaning from cardiopulmonary bypass (CPB) was not possible (post-cardiotomy ECMO). Due to favorable results (1), the indications have been extended to provide therapy to patients with cardiogenic shock requiring massive inotropic support, and finally for out of hospital cardiac arrest scenarios (2). For all of these indications, a cardiopulmonary support in the form of venoarterial (VA) ECMO is required. The ability of VA ECMO to provide cardiac and respiratory support differs from the 
isolated oxygenation-ventilation support provided by venovenous (VV) ECMO. To qualify for VV ECMO support, the cardiac pump function must be adequate to maintain pulmonary and systemic perfusion. When possible, echocardiography is performed to assess systolic ventricular function to aid decision-making.

The most common indications for ECMO implantations are listed in Table 1. The list of contraindications for ECMO in children is shrinking, with absolute contraindications including lethal chromosomal or syndromic abnormalities, and severe, irreversible brain or multiorgan injury.

\section{ECMO circuit and implantation technique}

In the pediatric ECMO population, we face a wide range of weights and dimensions ranging from 4.25 to $60 \mathrm{~kg}$. Therefore, different ECMO circuits and cannulas catering to these weight categories are used. As previously published (3), we use a Thoratec Centrimag ${ }^{\circledR}$ or PediVas $^{\circledR}$ (Levitronix, Zurich, Switzerland) console with a back-up unit, heater unit, and a Sechrist Air-Oxygen-Mixer (Sechrist Industries, Anaheim, USA). For neonates and infants weighing up to $15 \mathrm{~kg}$, the whole circuit contains approximately $250 \mathrm{~mL}$ priming volume with flow ranges up to $1.7 \mathrm{~L} / \mathrm{min}$; for children above $15 \mathrm{~kg}$, the volume of priming solution is about $750 \mathrm{~mL}$, with a capacity of achieving flow rates up to $7.0 \mathrm{~L} / \mathrm{min}$, which can also sufficiently cater to adult patients. Likewise, implantation sites differ. In adults, and in an emergency setting like cardiac arrest, femoral access is mainly used. In children, especially before the walking age, as a rule, the femoral vessels are not developed well enough to be suitable for ECMO cannulation and support. Cannulation for neonates, infants and small children focuses on either the neck vessels or large central vessels via median sternotomy. Neck cannulation has the advantage of being an expeditious procedure in an emergency situation, even during mechanical CPR, without causing major interruption to chest compressions. On the other hand, higher flow rates on ECMO might be able to be achieved when using the ascending aorta and the right atrium as cannulation sites. Theoretically, neck cannulation may carry the risk of blocking antegrade flow to the RCA, which in the setting of CPR, cerebral ischaemia or an incomplete circle of Willis, may increase the risk of ischaemic or haemorrhagic stroke. However, there is no clear evidence in the literature for this assumption (4). For VV ECMO implantations, our primary cannulation strategy is the internal jugular vein using the Avalon cannula (AVALON ${ }^{\circledR}$, Avalon Laboratories, LLC, California, USA). We have performed both surgical cut down to the vessel, as well as cannulation using the Seldinger technique for implantation. Implantation is guided by either echocardiographic or X-ray imaging to ensure adequate orientation of the cannula ports. Their proper position must be confirmed with echocardiography, especially the location of the outflow lumen, which must be positioned in the center of the right atrium with the flow directed towards the tricuspid valve. More "traditional" implantation sites combine the jugular vein and femoral vein. Advantages of a dual-lumen cannula using a single implantation site include a decrease in blood recirculation, easier patient mobilization, and lower risk of dislodgment (5).

Different combinations of the implantation sites are possible (i.e., internal carotid artery and right atrium), but other sites may also be used in older children, such as the subclavian artery and femoral vessels as in adults.

\section{ECMO in the setting of cardiac arrest}

Patients in cardiac arrest refractory to CPR may be placed on ECMO (commonly known as eCPR). The application of eCPR seems to provide superior cerebral perfusion compared to external compressions $(6,7)$. The reported incidence of cardiac arrest after cardiac surgery varies between $0.7-8 \%$ (8). ECPR remains an integral part of every "state of the art" congenital cardiac surgery program. As per published guidelines, it is recommended that post-cardiotomy patients should undergo re-sternotomy within 5 minutes of cardiac arrest (9). If no reversible cause of cardiac arrest such as pericardial tamponade is found, ECMO implantation should be performed. In cardiac arrest scenarios with in- or out-of-hospital CPR, all efforts must be made to minimize the time to ECMO implantation. There is inadequate clarity regarding criteria such as duration to $\mathrm{ECMO}$, lowest $\mathrm{pH}$, highest lactate and time taken for $\mathrm{pH}$ to normalize in relation to clinical and neurological outcomes, which would guide us in optimizing use of this critical resource intensive therapy. The decision as to whether eCPR is provided or not is mainly based on institutional algorithms (10).

Nevertheless, CPR duration $>30$ minutes was reported to be a significant risk factor for death (11). According to 


\begin{tabular}{|c|c|c|}
\hline Indications & Neonate & Pediatric \\
\hline \multirow[t]{3}{*}{ Cardiac } & $\begin{array}{l}\text { Congenital defect: } \\
\text { * Hypoplastic left heart syndrome; } \\
\text { * Left ventricular outflow obstruction; } \\
\text { * Septal defects }\end{array}$ & $\begin{array}{l}\text { Congenital defect: } \\
\text { * Left ventricular outflow obstruction; } \\
\text { * Right ventricular outflow obstruction; } \\
\text { * Septal defects }\end{array}$ \\
\hline & $\begin{array}{l}\text { Cardiomyopathy (bridge to recovery, transplant or long- } \\
\text { term MCS) }\end{array}$ & $\begin{array}{l}\text { Cardiomyopathy (bridge to recovery, transplant or long- } \\
\text { term MCS) }\end{array}$ \\
\hline & Myocarditis & Myocarditis \\
\hline \multirow[t]{5}{*}{ Respiratory } & Meconium aspiration syndrome & Pneumonia (viral/bacterial/aspiration) \\
\hline & $\begin{array}{l}\text { Persistent pulmonary hypertension of newborn/persistent } \\
\text { fetal circulation }\end{array}$ & Acute respiratory distress syndrome \\
\hline & Respiratory distress syndrome & \\
\hline & Congenital diaphragmatic hernia & \\
\hline & $\begin{array}{l}\text { Pneumonia (viral/bacterial/aspiration) } \\
\text { Sepsis }\end{array}$ & \\
\hline \multirow[t]{5}{*}{$\begin{array}{l}\text { Other indications } \\
\text { at any age }\end{array}$} & $\begin{array}{l}\text { Transplantation: } \\
\text { * Pre-transplantation as bridge to Tx; } \\
\text { * Primary graft dysfunction after heart or lung Tx }\end{array}$ & \\
\hline & $\begin{array}{l}\text { Elective periprocedural support: } \\
* \text { During lung transplantation or tracheal surgery }\end{array}$ & \\
\hline & $\begin{array}{l}\text { Cardiac arrest from any cause: } \\
\text { Bridge to decision; } \\
* \text { Underlying treatable disease }\end{array}$ & \\
\hline & Air leak syndrome & \\
\hline & Failure to wean from CPB & \\
\hline
\end{tabular}

ECMO, extracorporeal membrane oxygenation; MCS, mechanical circulatory support; Tx, transplant; CPB, cardiopulmonary bypass.

the latest pediatric ELSO report, the majority of children (73\%) received CPR for less than 60 min before ECMO support (12). Regular staff education and cardiac arrest simulations including check-lists which defines task allocation of each participant during eCPR may facilitate the intervention and prevent unnecessary collisions during CPR (10).

The use of eCPR is increasing in children (12). The 2016 ELSO report shows that almost $11 \%$ of all ECMO implantations are done in the setting of cardiac arrest (1). Thirteen percent of all pediatric ECMO cases are initiated in cardiac arrest scenarios, with the highest percentage being neonates (36\% of all neonatal ECMO implantations) (1). While eCPR is typically performed via the femoral vessels in adults, this is not a valid option in children and neonates. As previously discussed, neck cannulation in pediatric patients has the advantage of allowing uninterrupted chest compressions during ECMO implantation, whereas during central cannulation, chest compressions must be interrupted for surgical placement. As outlined above, however, an important issue to consider with neck cannulation in children is that a sufficient cerebral perfusion depends on a functioning circle of Willis. Some centers ligate the distal internal carotid artery to protect from thromboembolism and subsequent cerebral infarction; others do not and reconstruct the internal carotid artery after cannula explantation. Long-term neurologic outcome in pediatrics after ECMO remains a matter of research. 
ECMO implantation (total)

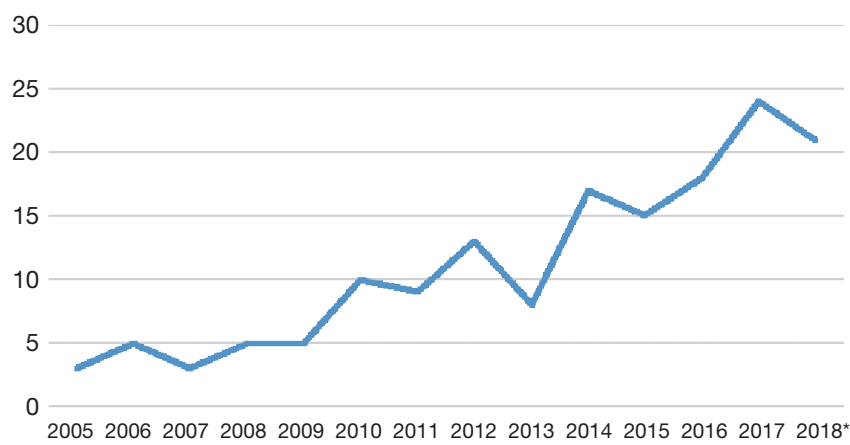

Figure 1 Absolute numbers of ECMO implantations at our unit. *, until 1st of July 2018. ECMO, extracorporeal membrane oxygenation.

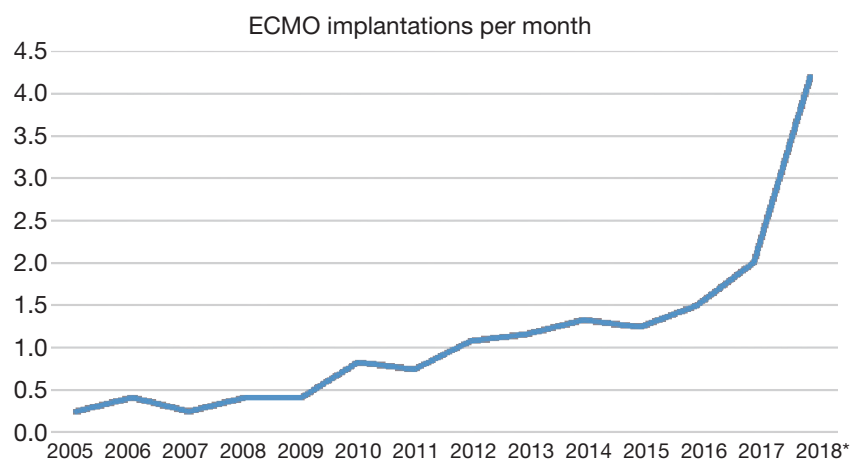

Figure 2 The distinct rise of ECMO implantations per month at our unit. *, until 1st of July 2018. ECMO, extracorporeal membrane oxygenation.

When central cannulation is performed, both internal carotid arteries remain perfused. Another advantage of central cannulation lies in the access and size of the target vessels. It can be presumed that central cannulation helps achieve higher flow rates as it enables the implantation of larger diameter cannulae.

Survival to discharge after eCPR varies between $29 \%$ to $43 \%$, depending on age group and whether in- or outof-hospital CPR was performed $(1,2,12,13)$. Prematurity represents an important risk factor in both non-CPR ECMO and eCPR scenarios (14). Data from the ELSO registry indicates that the overall survival rate to discharge for premature infants ( $<37$ weeks gestation) who received cardiac extracorporeal life support (ECLS) was $31 \%$, compared to $41 \%$ in term infants (1). Similarly, while the reported survival in pre-term babies following eCPR has approached $30 \%$, it can be as low as $21 \%$ in neonates
$<34$ weeks gestational age.

\section{Own demographic data}

Over the past years, we have observed a substantial increase in the number of ECMO implantations (see Figure 1). While there were 0.25 to 1.1 implants per month between 2005 and 2013, we saw a significant increase of up to four implants per month in 2018 (see Figure 2). Since 2014, our team at the children' hospital in Zurich has performed 92 ECMOs implantations in 34 neonates and 58 pediatric patients (see Table 2). The majority (80\%) were (VA) ECMO placements, while $20 \%$ were VV implants (Table 3). The primary cannulation strategy for all ECMO placements is shown in Table 4. Sternotomy with central cannulation was used in $54 \%$ of patients, followed by neck cannulation (42\%) and others (3\%). All VV ECMO were inserted via the internal jugular vein using the Avalon cannula (AVALON ${ }^{\circledR}$, Avalon Laboratories, LLC, California, USA). Surgical cutdown was performed in 15 cases, whereas in three cases the jugular vein was punctured using a Seldinger technique. The smallest child receiving VV ECMO support using Avalon cannula was 50 days old and weighted 4.2 kilograms.

Out of 92 implants, 29 placements (32\%) have been performed in the setting of cardiac arrest. All eCPR implants were VA ECMO with either neck $(n=8)$ or central cannulation $(\mathrm{n}=21)$ (Table 3). Eleven implants have been done in the operating theatre (OR), whereas the majority (90\%) has been installed outside the OR. Survival to discharge out of hospital was $28 \%$, with a higher likelihood in the pediatric group ( 0.35 per patient) compared to neonates (0.1 per patient) (Table 5). For this report, we did not investigate differences between in-hospital CPR and out-of-hospital CPR, which is a known risk factor for outcome (2).

\section{Long-term outcome}

Most of the reports reviewed have focused on outcomes until hospital discharge or transfer. Long-term outcome and further development are not well reported, for a number of reasons. First, it is clear that the outcome depends on the underlying disease and indication for ECMO. While children recovering from respiratory failure or sepsis might have a normal life expectancy, children suffering from complex congenital heart disease might require many re-do surgeries and a limited life expectancy. In The Netherlands, Hanekamp and colleagues evaluated national wide newborns 


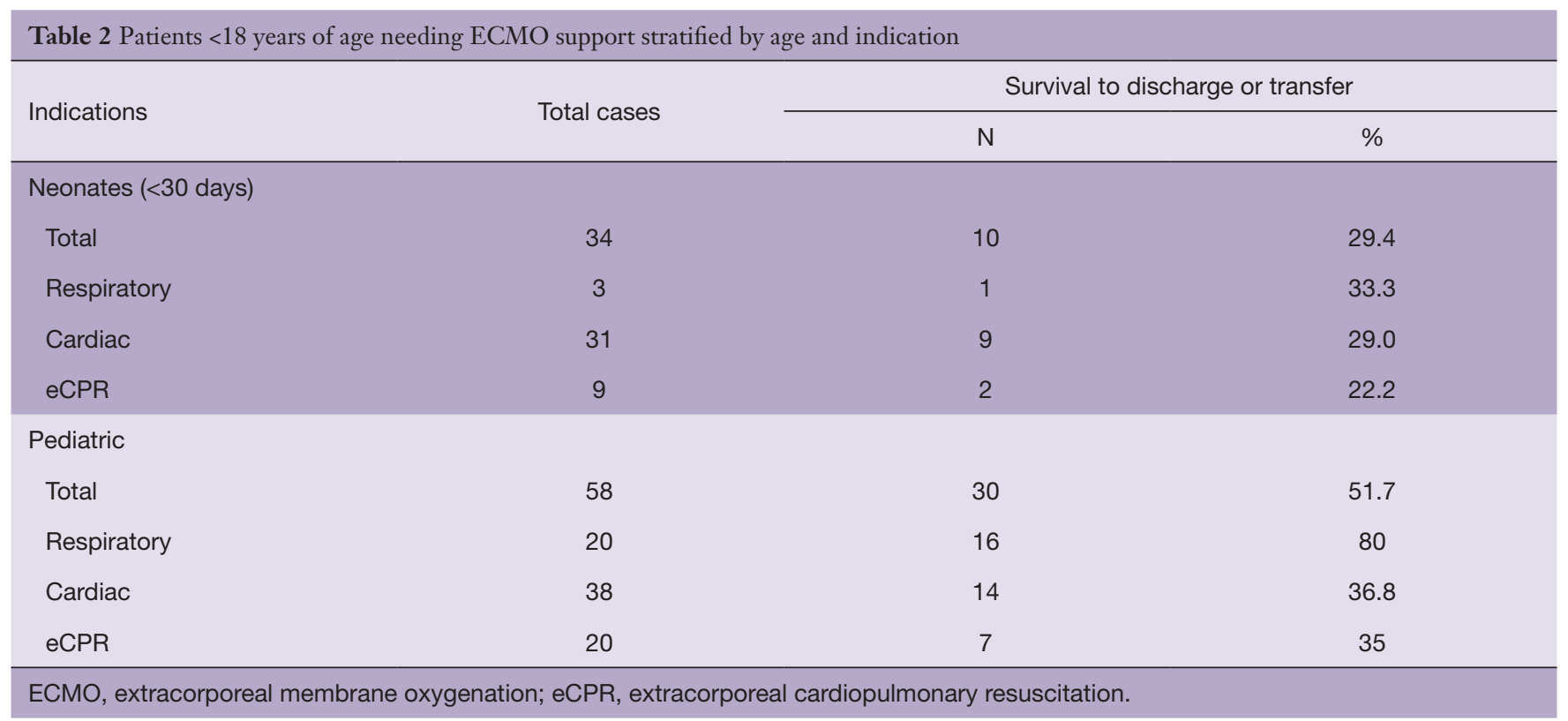

\begin{tabular}{lc}
$\begin{array}{l}\text { Table } 3 \text { ECMO strategies in patients }<18 \text { years of age needing } \\
\text { ECMO support }\end{array}$ \\
\hline ECMO strategies & 92 \\
\hline ECMO (overall) & 18 \\
\hline VV ECMO* & 74 \\
\hline VA ECMO* & 29 \\
\hline ECMO under CPR (eCPR) & 92 , \\
\hline *, until 1st of July 2018. ECMO, extracorporeal membrane \\
$\begin{array}{l}\text { oxygenation; VV, veno-venous; VA, veno-arterial; CPR, cardio } \\
\text { pulmonary resuscitation; eCPR, extracorporeal CPR. }\end{array}$
\end{tabular}

treated with ECMO at 5 years of age and focused on cognitive, neuromotor, and neuropsychological outcomes. A small percentage (6\%) suffered major neurodevelopmental disability, but overall mean cognitive development did not differ significantly from the control group (15). More research is necessary to address the long-term neurological outcome in children receiving ECMO stratified by variables including indications, type of cannulation, type of ECMO and duration of CPR.

\section{Comments}

Traditionally, ECMO has a high value in pediatrics and neonates, and implantation numbers have been increasing

\begin{tabular}{|c|c|}
\hline Cannulation strategy & Implantation, $\mathrm{N}$ \\
\hline \multicolumn{2}{|l|}{ Neck cannulation } \\
\hline VA & 21 \\
\hline VV & 18 \\
\hline \multicolumn{2}{|l|}{ Chest cannulation } \\
\hline VA & 50 \\
\hline WV & 0 \\
\hline \multicolumn{2}{|l|}{ Other } \\
\hline VA & 3 \\
\hline VV & 0 \\
\hline \multicolumn{2}{|l|}{ Cannulation in eCPR ${ }^{*}$} \\
\hline Neck & 8 \\
\hline Chest & 21 \\
\hline \multicolumn{2}{|c|}{$\begin{array}{l}\text { *, until 1st of July 2018. ECMO, extracorporeal membrane } \\
\text { oxygenation; VV, veno-venous; VA, veno-arterial; eCPR, } \\
\text { extracorporeal cardiopulmonary resuscitation. }\end{array}$} \\
\hline
\end{tabular}

over the past few decades. ECMO can be used in many settings to support pediatric patients with cardiac, respiratory, and other indications. In light of the growing applications of ECMO, however, a detailed analysis of outcomes is 


\begin{tabular}{|c|c|c|c|}
\hline \multirow[t]{3}{*}{ Neonates $(<30$ days) $(n=9)$} & Respiratory & 0 & 0 \\
\hline & Postcardiotomy* & 4 & 0 \\
\hline & Cardiac-non-postcardiotomy & 5 & 1 \\
\hline \multirow{2}{*}{ Pediatric $(n=20)$} & Postcardiotomy* & 8 & 4 \\
\hline & Cardiac-non postcardiotomy & 10 & 2 \\
\hline
\end{tabular}

becoming increasingly important to ensure that this resourceintensive therapy remains available for appropriate candidates and facilitates favorable long-term survival and functional prognosis. In particular, greater research is required to investigate long-term neurodevelopmental outcomes in surviving pediatric ECMO patients.

\section{Acknowledgements}

None.

\section{Footnote}

Conflicts of Interest: The authors have no conflicts of interest to declare.

\section{References}

1. Thiagarajan RR, Barbaro RP, Rycus PT, et al. Extracorporeal Life Support Organization Registry International Report 2016. ASAIO J 2017;63:60-7.

2. Wang CH, Chen YS, Ma MH. Extracorporeal life support. Curr Opin Crit Care 2013;19:202-7.

3. Schmiady M, Döll C, Cavigelli-Brunner A, et al. Extracorporeal membrane oxygenation in neonates and children. Cardiovascular Medicine 2017;20:57-61.

4. Kurkluoglu M, Hynes CF, Alfares FA, et al. Choice of Peripheral Venoarterial Extra-Corporeal Membrane Oxygenation Cannulation Site in Patients Above 15 kilograms. J Card Surg 2015;30:461-5.

5. Rubino A, Vuylsteke A, Jenkins DP, et al. Direct complications of the Avalon bicaval dual-lumen cannula in respiratory extracorporeal membrane oxygenation
(ECMO): Single-center experience. Int J Artif Organs 2014;37:741-7.

6. Martin GB, Rivers EP, Paradis NA, et al. Emergency department cardiopulmonary bypass in the treatment of human cardiac arrest. Chest 1998;113:743-51.

7. Nagao K, Hayashi N, Kanmatsuse K, et al.

Cardiopulmonary cerebral resuscitation using emergency cardiopulmonary bypass, coronary reperfusion therapy and mild hypothermia in patients with cardiac arrest outside the hospital. J Am Coll Cardiol 2000;36:776-83.

8. Society of Thoracic Surgeons Task Force on Resuscitation After Cardiac Surgery. The Society of Thoracic Surgeons Expert Consensus for the Resuscitation of Patients Who Arrest After Cardiac Surgery. Ann Thorac Surg 2017;103:1005-20.

9. Truhláŕ A, Deakin CD, Soar J, et al. European Resuscitation Council Guidelines for Resuscitation 2015: Section 4. Cardiac arrest in special circumstances. Resuscitation 2015;95:148-201.

10. Erek E, Aydin S, Suzan D, et al. Extracorporeal cardiopulmonary resuscitation for refractory cardiac arrest in children after cardiac surgery. Anatol J Cardiol 2017;17:328-33.

11. Tajik M, Cardarelli MG. Extracorporeal membrane oxygenation after cardiac arrest in children: what do we know? Eur J Cardiothorac Surg 2008;33:409-17.

12. Barbaro RP, Paden ML, Guner YS, et al. Pediatric Extracorporeal Life Support Organization Registry International Report 2016. ASAIO J 2017;63:456-63.

13. Richardson AS, Schmidt M, Bailey M, et al. ECMO Cardio-Pulmonary Resuscitation (ECPR), trends in survival from an international multicentre cohort study over 12-years. Resuscitation 2017;112:34-40. 
14. Di Nardo M, MacLaren G, Marano M, et al. ECLS in Pediatric Cardiac Patients. Front Pediatr 2016;4:109.

15. Hanekamp MN, Mazer P, van der Cammen-van Zijp MH,

Cite this article as: Erdil T, Lemme F, Konetzka A, CavigelliBrunner A, Niesse O, Dave H, Hasenclever P, Hübler M, Schweiger M. Extracorporeal membrane oxygenation support in pediatrics. Ann Cardiothorac Surg 2019;8(1):109-115. doi: 10.21037/acs.2018.09.08 et al. Follow-up of newborns treated with extracorporeal membrane oxygenation: a nationwide evaluation at 5 years of age. Crit Care 2006;10:R127. 